\title{
A Control Strategy Design Applied To Single-Phase Grid-Connected Inverters
}

\author{
E.N.Chaves ${ }^{1}$, E.A.A.Coelho ${ }^{2}$, G.P.Viajante ${ }^{1}$, L.C.G.Freitas ${ }^{2}$, L.G.Wezs ${ }^{1}$, L.C.Freitas ${ }^{2}$, C.A.Queiroz ${ }^{1}$ and \\ V.R,Bernadeli ${ }^{1}$. \\ ${ }^{1}$ Federal Institute of Education, Science and Technology of Goiás \\ Campus of Itumbiara - Goiás, (Brazil) \\ Phone/Fax number: 5564 21035600, e-mail: A. ghunterp@gmail.com and eric.neryx@gmail.com \\ ${ }^{2}$ Federal University of Uberlandia \\ Campus Santa Mônica \\ Phone/Fax number: 5534 3239-4411
}

\begin{abstract}
This paper presents the design of the One-Degree of Freedom Internal Model Control structure (1-DOF IMC) applied to a single-phase grid-connected inverter. It is presented the mathematical model of the inverter and its LCL filter, used to project the 1-DOF IMC controller, and it is analyzed the internal feedback compensation corresponding to the decoupling of grid voltage provided by a feedforward strategy. In order to establish a comparison base, the grid connected system is also evaluated using a Proportional-Resonant Controller (P-Res). Simulations results of both controllers are presented, which allows the comparison between the 1-DOF IMC and P-Res control performances.
\end{abstract}

\section{Key words}

Grid Connected Single-Phase Systems, Internal Model Control, Photovoltaic Generation, Proportional-Resonant Controller.

\section{Introduction}

The increasing demand for electric power in recent years culminated in new researches on renewable power sources such as wind and photovoltaic (PV). In Brazil, two main points justify the research and development on PV systems, first is the high level of diary solar irradiance, which can exceed $5 \mathrm{kWh} / \mathrm{m}^{2}$ in some areas [1]. Second, the necessity to meet the demand for power with fewer losses and cost reduction in electrical transmission and distribution, since the power system is operating on its technical limits.

Many researches regarding electric power generation from solar panels have been conducted and, in general, they are focused on efficiency improvements through means of Maximum Power Point Tracking (MPPT) algorithms [2], for example, or improving the quality of injected power, for grid tie systems [3].

In grid-connected Distributed Generation systems (DG), issues involving the design criteria or topology have attracted great interest by the scientific community and have increased the number of publications, among which [4] and [5] respectively stand out. The topology adopted for this work was the single-phase PWM sinusoidal Voltage Source Inverter (VSI) with LCL filter, connected to a single-phase grid [5]. The structure is presented in Figure 1.

The DC bus voltage control will be provided by an external loop with PI compensator, which defines the reference for the current to be injected into the grid.

In the case of control strategies for the current to be injected into the grid, the development of the first applied controllers in VSIs started with Hysteresis and Proportional-Integral (PI) types. These controllers are able to compensate such grid-connected power injection systems if used in three-phase topologies with a synchronous reference. Otherwise, they cannot compensate the steady-state errors, in terms of amplitude and phase of the injected current [6]. However, for stationary referential - the case of single-phase applications - worth noting advances were described in [6], Proportional-Resonant (P-Res), and in [7], the Repetitive Controller.

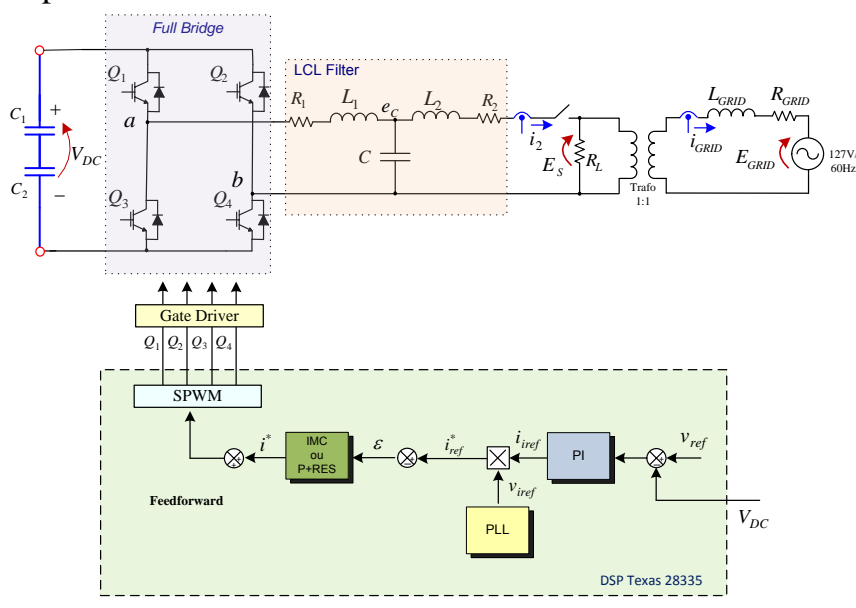

Fig. 1. Structure of the Single-Phase Grid-Connected System.

This paper presents a control strategy for active power injection into an electric grid with similar performance of the P-Res controller, regarding steady-state errors. This performance was possible with the development of structure 1-DOF IMC and the feedforward strategy provided by the grid voltage decoupling. 
To validate the control strategy presented herein, a Type II P-Res Compensator, [6] e [8], was designed for purposes of comparison with 1-DOF IMC. The results are presented and discussed.

\section{The Feedforward Strategy}

This Feedforward strategy, sometimes called BackEMF [9] by being similar to back counter-electromotive force feedback of DC current machines control, consists on cancellation of the grid voltage negative feedback, which is intrinsic from inverter system. This method, already well explored in [9], [10] and [11], has the effect of decoupling the local load $\left(R_{L}\right)$ voltage, which is also the grid voltage (considering $Z_{G R I D}=s L_{G R I D}+R_{G R I D}$ tending to zero and $E_{S}$ tending to $\left.E_{G R I D}\right)$ at the connection point, from a sample of that voltage added to the current injection control action $\left(u_{\text {cont }}\right)$ before the actuator, in this case, the PWM generator. Figure 2 shows the diagram that represents the process model, which integrates the IGBT's bridge and the LCL output filter.

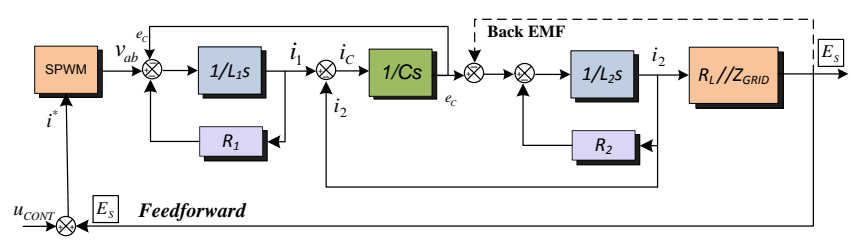

Fig. 2. State diagram for the inverter connected to the grid and the effect due to the Feedforward strategy.

\section{The Voltage Inverter Modeling}

The modeling of the inverter and the LCL output filter was made using the Average State Space technique [12] [13], taking into account two switching intervals with the respective resulting circuits and the grid voltage decoupling.

And so,

$$
G_{i 2 / d}=\hat{i}_{2}(s) / \hat{d}(s)=\frac{2 V_{a b} k}{b s^{3}+c s^{2}+d s+e}
$$

in which:

$$
\begin{gathered}
b=C L_{1} L_{2} \\
c=C L_{1} R_{2}+C L_{2} R_{1} \\
d=C R_{1} R_{2}+L_{1}+L_{2} \\
e=R_{1}+R_{2} .
\end{gathered}
$$

Therefore, $\left(G_{i 2 / d}\right)$ is the transfer function for smallsignal considering the output changing in $i_{2}$ (the output current) as function of the PWM active cycle width $(\hat{d})$. The term $k$ is a constant of proportionality to make the
PWM (actuator) gain unitary, and $V_{a b}$ is the average value of the voltage at the point of connection between the fullbridge output and the LCL filter input.

\section{The Internal Model Based Controller}

The term Model Based Control - MBC is used to designate the control systems that explicitly incorporates a process model in the algorithm, in particular the control methods such as Internal Model Control (IMC) and the Model Predictive Control - (MPC). Figure 3 illustrates in a generic way the 1-DOF IMC based control strategy, in which $r(s)$ is the reference, $y(\mathrm{~s})$ is the process output, $p(s)$ is the process,$\tilde{p}(s)$ is the process model,$u(s)$ is the control effort, $d(s)$ is the disturbance and $\tilde{d}_{\varepsilon}(s)$ is the estimated disturbance [14].

Since $\tilde{p}(s)$ is a perfect representation of the process $(p(s))$, if the controller gain $(q(s, \varepsilon))$ is the reciprocal of the model gain, the output $(y(s))$ will achieve the reference $(r(\mathrm{~s}))$, on condition that the process and the model gains have the same signal and the controller is tuned so that the stability is ensured.

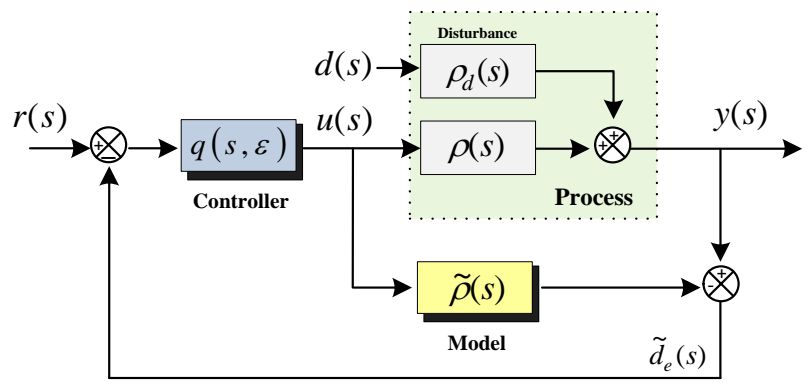

Fig. 3. 1-DOF IMC based structure.

In order to get the transfer functions of $d(s), r(s)$ and $y(s)$ and understand the operation of this control strategy, Figure 3 was redesigned and presented in Figure 4 like a feedback system with traditional structure in which:

$$
c(s) \equiv \frac{u(s)}{e(s)}=\frac{q(s)}{1-q(s) \tilde{p}(s)}
$$

Being $c(s)$ the control action, $u(s)$ the controller effort and $e(s)$ the error.

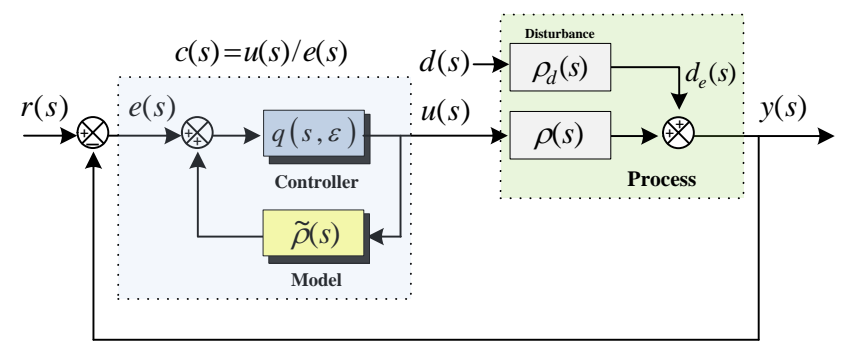

Fig. 4. 1-DOF IMC reorganized structure. 
From the relations between the input and the output diagramed in Figure 4, one can deduce that:

$$
y(s)=\frac{p(s) q(s) r(s)}{1+(p(s)-\tilde{p}(s)) q(s)}
$$

And,

$$
y(s)=\frac{(1-\tilde{p}(s) q(s)) p_{d}(s) d(s)}{1+(p(s)-\tilde{p}(s)) q(s)}
$$

In which $p_{d}(\mathrm{~s})$ is the system model due to the disturbance, thus, the effect of the disturbance flows through the system and adds to the output.

Applying the final value theorem, if the equations (7) and (8) are dynamically stable and the controller gain $q(0)$ is chosen to be the reciprocal of the internal model gain, then $\tilde{p}(0) q(0)=1$ and the gain of the denominator of (7) and (8) will be $p(0) q(0)$. Thus, the forward path gain is unitary, the gain between the disturbance $d(s)$ and $y(s)$ is zero and the steady state error is zero. Therefore, the idea behind this strategy is to achieve:

$$
y(s)=r(s) \quad \text { and } \quad y(s) / d(s)=0
$$

Provided that:

$$
p(s) q(s)=1 \quad \text { and } \quad \tilde{p}(s)=p(s)
$$

Therefore, for the control action be effective, a perfect model is necessary and the controller has to invert such model [14]. However, in practice, it is impossible to have a perfect model and if it has any dynamics (very common), none controller can invert the process in the exact way. How close it can get, depends on its design.

\section{A. The 1-DOF IMC Controller Design}

Using the criteria discussed in [14] for the designing when the process transfer function does not have zeros close to the imaginary axis neither on the right side in the s-plane, the controller can be designed as:

$$
q(s)=\frac{D(s)}{N(s) \cdot(\varepsilon s+1)^{r}}
$$

In which $N(s)$ and $D(s)$ are the numerator and the denominator, respectively, of the transfer function (1) already described, and $r$ is defined to be the difference between the denominator order and the numerator order of the transfer function.

The expression (12) represents the filter term that makes $q(s)$ causal (and physically feasible), and $\varepsilon$ is an arbitrary parameter that defines the filter cutoff frequency.

$$
\frac{1}{(\varepsilon s+1)^{r}}
$$

In order to maintain the committed relationship between the inverter dynamics, in terms of the velocity of response in the current imposition, and avoid the resonance frequency $\left(f_{\text {res }}\right)$ of $L_{l}$ and $C$, besides the effect of the PWM switching, the value of the parameter $\varepsilon-$ determined interactively - was fixed in 0.0003 . Above this value the systems becomes slow and the phase lagging between the reference and the current injected becomes pronounced. Below this value, the resonance effect at $876 \mathrm{~Hz}$ becomes larger.

Figure 5 illustrates the Bode Plot for $p(s)$ - inverter, $q(s)$ - controller and $g(s)=p(s) q(s)$ - the forward path gain. One can note that inside the pass band of $g(s)$ the gain is unitary $(0 \mathrm{~dB})$, in accordance with (9) and (10). The controller just cannot completely invert the response because of the filter, modeled in (12), which keeps the flat response after $f_{\text {res }}$, wherein the gain amplitude is defined by $\varepsilon$, as described in [14]. The resulting effect in $g(s)$ is a low-pass filter with a pass band of $475 \mathrm{~Hz}(3 \mathrm{~dB}$ frequency), however, without the resonance peak which is canceled by the controller, damping the system. There is also a mitigation and almost linearization of the phase angle, especially around $f_{\text {res }}$, which can be inferred from Figure 5.

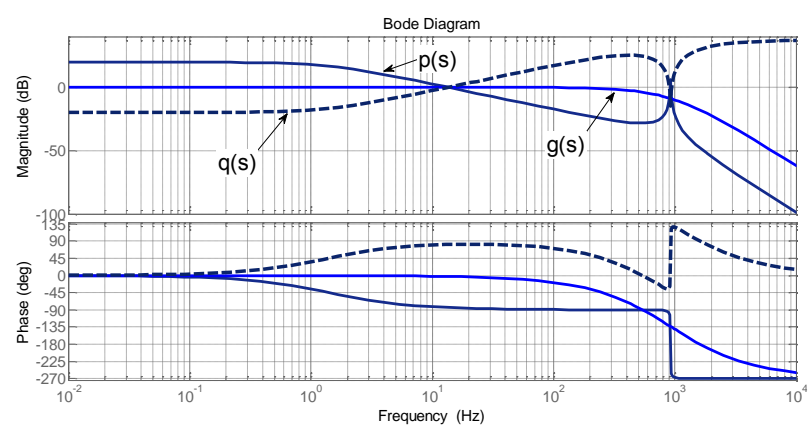

Fig. 5. Frequency response of the inverter, the 1-DOF IMC controller and the forward path gain.

\section{The P-Res Controller Design}

For the P-Res control strategy tested in this work, it was chosen the transfer function defined by (13) and refered in [6] as the Type II controller, whose transfer function is:

$$
G_{r e s}=k p+\frac{2 k i \omega_{c} s}{s^{2}+2 \zeta \omega_{c} s+\omega_{c}^{2}}
$$

Where $K p$ and $K i$ are the proportional and integral gains, respectively, $\zeta$ is the damping ratio and $\omega_{c}$ is the centre frequency. The design options (type, damping ratio and centre frequency) for the controller will not be detailed, since they were explored in [6] and [8], and they are not the focus of this paper. 
However, the parameters used in this design, which were determined by trial and error, searching for the best result, can be seen at Table I.

TABLE I - P-Res Parameters

\begin{tabular}{ccc}
\hline Parameter & Name & Value \\
\hline Proportional Gain & $K p$ & 0.7 \\
\hline Integral Gain & $K i$ & 3.0 \\
\hline Damping Ratio & $\zeta$ & 0.03 \\
\hline Centre Frequency & $\omega_{c}$ & $2 \pi 60$ \\
\hline
\end{tabular}

Therefore, the controller transfer function defined in (13) with the parameters of Table I presents a frequency response of a band-pass filter with center frequency equals to the grid frequency. The open-loop frequency response for the system with the P-Res controller is shown in Figure 6.

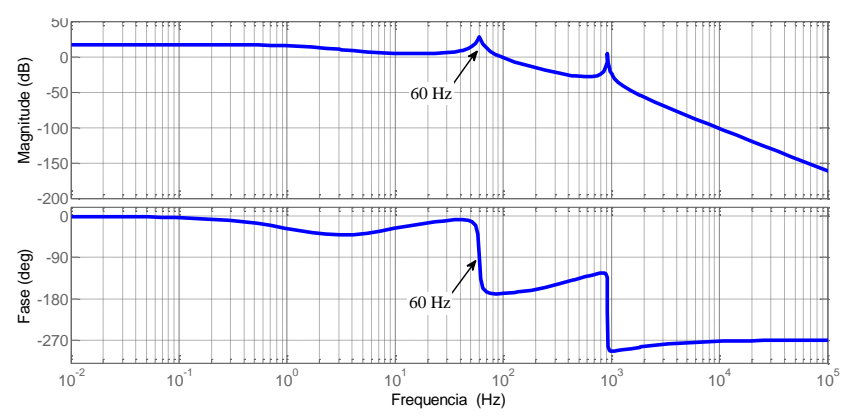

Fig. 6. P-Res Type II frequency response.

\section{Simulation Results}

Computer simulations of the injection of active power into a single-phase grid using 1-DOF IMC and P-Res were done, aiming to comparatively evaluate their performance. The P-Res was tested with and without Feedforward.

Figure 7 shows the results using P-Res without Feedforward. In Figures 7, 8 and 9 I-L2 represents the inverter output current $I_{2}$ (which flows through the connection inductance) and $I_{G R I D}$ is the current supplied or received by the grid (after the connection point and the local load). The current consumed by the local load is the subtraction $I_{2}-I_{G R I D}$.

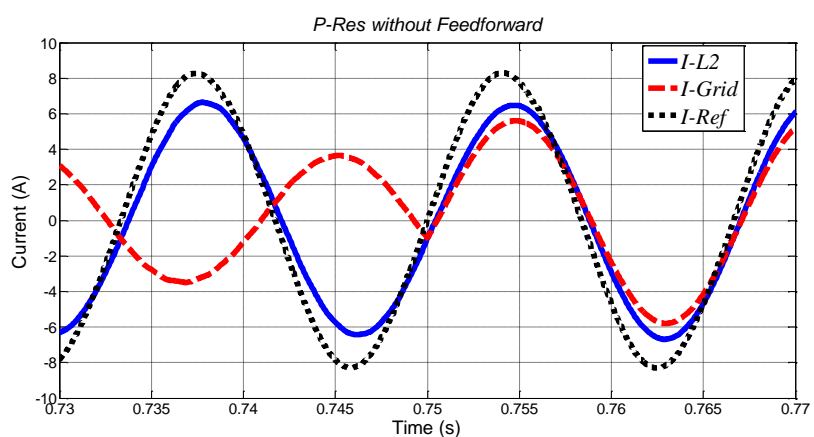

Fig. 7. Simulation results of the current injection with P-Res without Feedforward.
Between 0.73s and 0.75s the local load impedance was lower (about $20 \Omega$ ) and it drained current from inverter and grid, which explains the phase shift of $180^{\circ}$ between them. After $0.75 \mathrm{~s}$ the load impedance is changed to 200 $\Omega$ and at that moment, the inverter starts supplying current for the local load and injects the remainder into the grid.

Figure 8 shows the results for P-Res with the Feedforward strategy. A significant improvement can be noted, with reduction of the steady-state error. However, it should be noted that those results are related to the controller as defined in (13) with the parameters from Table I and may differ if the design criteria is different from those adopted here.

The 1-DOF IMC results are presented in Figure 9. One can note a very similar result obtained with the P-Res controller with Feedforward, i.e. small error at steady state and unitary power factor.

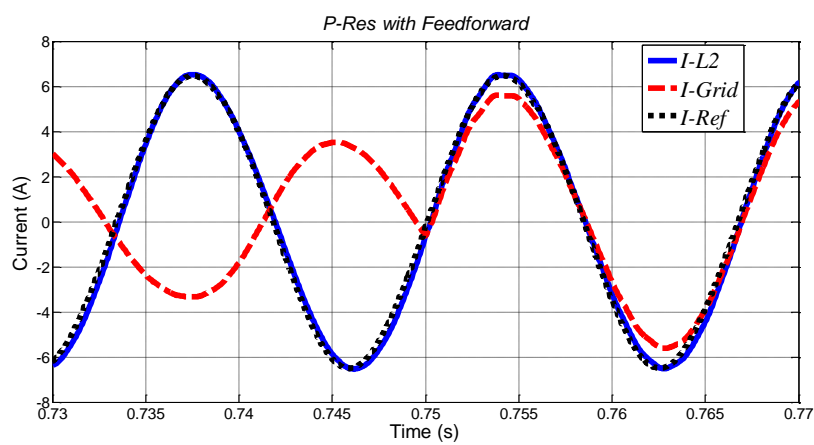

Fig. 8. Simulation results of current injection using P-Res with Feedforward.

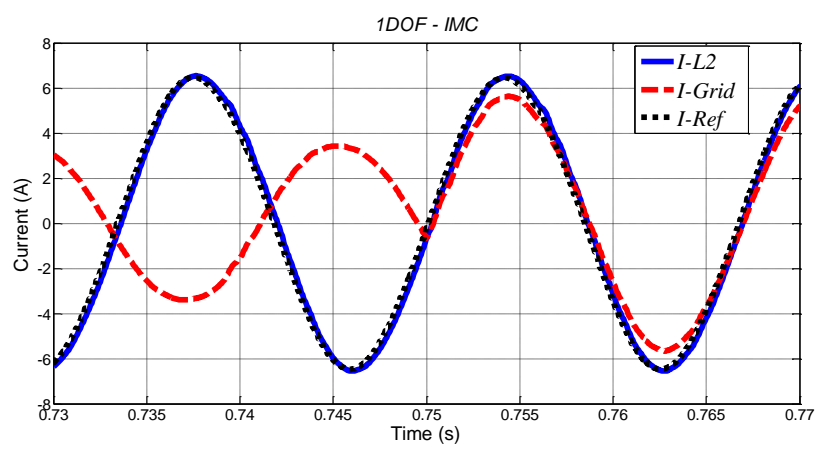

Fig. 9. Simulation results of current injection using 1-DOF IMC.

\section{Conclusion}

This paper presented the design stages of a 1-DOF IMC controller with application of the Feedforward strategy, for the control of current injection from a PWM Voltage Source Inverter tied to a single-phase power grid, through a LCL filter. This system was designed to be able to control the power injection from a photovoltaic array to the electrical grid, with similar performance when done using P-Res. The results for 1-DOF IMC although satisfactory in the case of steady-state error in 
module and phase synchronism with the grid voltage presented a slightly worse THD than the other. However, in a large range of operation, the 1-DOF IMC THD values changed less and the worst result remained within the accepted limits by international rules.

Therefore, the aim of this work was achieved successfully, the design steps were presented and the simulation results corroborate the quality and feasibility of the 1-DOF IMC + Feedforward controller for the power injection into the grid and, especially, confirms its suitability for systems in which the power availability changes much, for example, in photovoltaic generators.

\section{Acknowledgement}

The authors would like to thank Capes, CNPq (process $n^{\circ}$ 406845/2013-1), Fapemig (Case no APQ - 01219-13), IFG and NUPSE for the financial support.

\section{REFERENCES}

[1] Agência Nacional de Energia Elétrica (ANEEL). Atlas de Energia Elétrica do Brasil, 2nd Ed., Brasília, 2005.

[2] M. A. G. de Brito, L. Galotto, L. P. Sampaio, et al., "Evaluation of the Main MPPT Techniques for Photovoltaic Applications", in IEEE Transactions on Industrial Electronics, vol. 60, pp. 1156$1167,2012$.

[3] W. Libo, Z. Zhengming, L. Jianzheng, "A SingleStage Three-Phase Grid-Connected Photovoltaic System With Modified MPPT Method and Reactive Power Compensation", in IEEE Transactions on Energy Conversion, vol. 22, pp. 881-886, 2007.

[4] IEEE Standard 1547-2003, IEEE Standard for Interconnection Distributed Resource with Electric Power Systems, 2003.

[5] S.B. Kjaer, J. Pedersen, F. Glaabjerg, "A Review of Single-Phase Grid-Connected Inverters for Photovoltaic Modules", in IEEE Transactions on Industry Applications, vol. 41, pp. 1292-1306, 2005.

[6] D.N. Zmood, D.G. Holmes, "Stationary Frame Current Regulation of PWM Inverters with Zero Steady-State Error", in IEEE Transactions on Power Electronics, vol. 18, pp. 814-822, 2003.

[7] M. Rashed, C. Klumpner, G. Asher, "Repetitive and Resonant Control for a Single-Phase GridConnected Hybrid Cascaded Multilevel Converter", in IEEE Transactions on Power Electronics, vol. 28, pp. 2224-2234, 2013.

[8] H. Cha, T.K. Vu, J.E. Kim, "Design and Control of Proportional-Resonant Controller Base Photovoltaic Power Conditioning System", in Proc. Energy Conversion Congress and Exposition - Conference IEEE, pp. 2198-2205, 2009.

[9] M.J. Ryan, W.E. Brumsickle, R.D. Lorenz, "Control topology options for single-phase UPS inverters", in IEEE Transactions on Industry Applications, vol. 33, pp. 493-501, 1997.
[10] W. Li, D. Pan, X. Ruan, X. Wang "A FullFeedforward Scheme of Grid Voltages for a Threephase Grid-connected Inverter with an LCL Filter", in Proc. Energy Conversion Congress and Exposition - Conference IEEE, pp. 96-103, 2011.

[11] T. Liu, X. Hao, X. Yang, M. Zhao, L. Xiong “A Novel Grid Voltage Feed Forward Control Strategy for Three-phase Grid-connected VSI with LCCL Filter", in Proc. Industrial Electronics - Conference IEEE International Symposium on, pp. 86-91, 2012.

[12] R. D. Middlebrook; S, J.A. Cuk "A General Unified Approach to Modelling Switching-Converter Power Stages", in Proc. of Power Eletronics Specialists Conference, pp. 1-6, 1976.

[13] R. W. Erickson, D. Maksimóvic, Fundamentals of Power Electronics, Second Edition, 2004.

[14] C. Brosilow, B. Joseph, Techniques of ModelBased Control, Prentice Hall, first Edition, New York, 2002.

[15] International Electrotechnical Commission, IEC 61000-3-2, Electromagnetic Compatibility (EMC) Limits for Harmonic Current Emissions, 1998 and Emendation A14, 2001.

[16] IEEE 519-1992, IEEE Recommended Practice and Requirements for Harmonic Control in Electric Power Systems, 1992. 